\title{
Higher-order symmetric duality for a class of multiobjective fractional programming problems
}

\author{
Gao Ying
}

Correspondence: gaoyingimu@163. com

Department of Mathematics, Chongqing Normal University, Chongqing 400047, China

\begin{abstract}
In this paper, a pair of nondifferentiable multiobjective fractional programming problems is formulated. For a differentiable function, we introduce the definition of higher-order $(F, \alpha, \rho, d)$-convexity, which extends some kinds of generalized convexity, such as second order F-convexity and higher-order $F$-convexity. Under the higher-order $(F, \alpha, \rho, d)$-convexity assumptions, we prove the higher-order weak, higher-order strong and higher-order converse duality theorems.

Mathematics Subject Classification (2010) 90C29; 90C30; 90C46.
\end{abstract}

Keywords: Higher-order symmetric duality, multiobjective fractional programming, higher-order $(F, a, \rho, d)$-convexity.

\section{Introduction}

Symmetric duality in nonlinear programming in which the dual of the dual is the primal was introduced by Dorn [1]. The notion of symmetric duality was developed significantly by Dantzig et al. [2], and the Wolfe dual models presented in [2]. Mond [3] presented a slightly different pair of symmetric dual nonlinear programs and obtained more generalized duality results than that of Dantzig et al. [2]. Mond and Weir [4] then gave another pair of symmetric dual nonlinear programs in which a weaker convexity assumption was imposed on involved functions. Later, Mond and Weir [5], Weir and Mond [6] as well as Gulati et al. [7] generalized single objective symmetric duality to multiobjective case.

Chandra et al. [8] first formulated a pair of symmetric dual fractional programs with certain convexity hypothesis. Pandey [9] introduced second-order $\eta$-invex function for multiobjective fractional programming problem and established weak and strong duality theorems. Yang et al. [10] discussed a class of nondifferentiable multiobjective fractional programming problems, and proved duality theorems under the assumptions of invex (pseudoinvex, pseudoincave) functions. Higher-order duality in nonlinear programs have been studied by some researchers. Mangasarian [11] formulated a class of higher-order dual problems for the nonlinear programming problem by introducing twice differentiable functions. Mond and Zhang [12] obtained duality results for various higher-order dual programming problems under higher-order invexity assumptions. Under invexity-type conditions, such as higher-order type I, higher-order pseudo-type I, and higher-order quasi-type I conditions, Mishra and Rueda [13] gave various duality results. Recently, Chen [14] also discussed the duality theorems under

(c) 2012 Ying; licensee Springer. This is an Open Access article distributed under the terms of the Creative Commons Attribution License (http://creativecommons.org/licenses/by/2.0), which permits unrestricted use, distribution, and reproduction in any medium, provided the original work is properly cited. 
higher-order $F$-convexity ( $F$-pseudo-convexity, $F$-quasi-convexity) for a pair of multiobjective nondifferentiable program. But, up to now, there is not sufficient literatures dealing with higher-order fractional symmetric duality.

In this paper, we first formulate a pair of nondifferentiable multiobjective fractional pro-gramming problems. For a differentiable function $h: R^{n} \times R^{n} \rightarrow R$, we introduce the definition of higher-order $(F, \alpha, \rho, d)$-convexity, which extends some kinds of generalized convexity, such as second order $F$-convexity in [15] and higher-order $F$-convexity in [14]. Under the higher-order $(F, \alpha, \rho, d)$ - convexity assumptions, we prove the higherorder weak, higher-order strong and higher-order converse duality theorems.

\section{Preliminaries}

Let $R^{n}$ be the $n$-dimensional Euclidean space and let $R_{+}^{n}$ be its non-negative orthant. The following conventions for vectors in $R^{n}$ will be used:

$$
\begin{aligned}
& x<y \quad \text { if and only if } \quad y-x \in \operatorname{int} R^{n} ; \\
& x \leq y \quad \text { if and only if } \quad y-x \in R_{+}^{n} \backslash\{0\} ; \\
& x \leqq y \quad \text { if and only if } \quad y-x \in R_{+}^{n} ; \\
& x \not y \quad \text { is the negation of } \quad x \leq y .
\end{aligned}
$$

For a real-valued twice differentiable function $h(x, y)$ defined on an open set in $R^{n} \times R^{m}$, denote by $\nabla_{x} h(\bar{x}, \bar{y})$ the gradient vector of $h$ with respect to $x$ at $(\bar{x}, \bar{y}), \nabla_{x x} h(\bar{x}, \bar{y})$ the hessian matrix with respect to $x$ at $(\bar{x}, \bar{y})$. Similarly, $\nabla_{y} h(\bar{x}, \bar{y}), \nabla_{x y} h(\bar{x}, \bar{y})$ and $\nabla_{y y} h(\bar{x}, \bar{y})$ are also defined.

Let $C$ be a compact convex set in $R^{n}$. The support function of $C$ is defined by

$$
s(x \mid C)=\max \left\{x^{T} y: y \in C\right\} .
$$

A support function, being convex and everywhere finite, has a subdifferential, that is, there exists a $z \in R^{n}$ such that

$$
s(y \mid C) \geqq s(x \mid C)+z^{T}(y-x), \forall x \in C .
$$

The subdifferential of $s(x \mid C)$ is given by

$$
\partial s(x \mid C)=\left\{z \in C: z^{T} x=s(x \mid C)\right\} .
$$

For a convex set $D \subset R^{n}$, the normal cone to $D$ at a point $x \in D$ is defined by

$$
N_{D}(x)=\left\{y \in R^{n}: y^{T}(z-x) \leqq 0, \forall z \in D\right\} .
$$

When $C$ is a compact convex set, $y \in N_{C}(x)$ if and only if $s(y \mid C)=x^{T} y$, or equivalently, $x \in \partial s(y \mid C)$.

Consider the following multiobjective programming problem $(\mathrm{P})$ :

$$
\text { Minimize } f(x) \text { subject to } g(x) \leqq 0, \quad x \in X,
$$

where $f: R^{n} \rightarrow R^{m}, g: R^{n} \rightarrow R^{l}$ and $X \subset R^{n}$. Denote by $S$ the set of feasible solutions of (P).

Definition 2.1. (a) A feasible solution $x_{0}$ is said to be an efficient solution of (P) if there is no other $x \in S$ such that $f(x) \leq f\left(x_{0}\right)$. 
(b) A feasible solution $x_{0}$ is said to be a properly efficient solution of (P) if it is an efficient solution of $(\mathrm{P})$, and there exists a real number $M>0$ such that for all $i \in\{1, \ldots, m\}$, $x \in S$, and $f_{i}(x)<f_{i}\left(x_{0}\right)$,

$$
f_{i}\left(x_{0}\right)-f_{i}(x) \leqq M\left(f_{j}(x)-f_{j}\left(x_{0}\right)\right)
$$

for some $j \in\{1, \ldots, m\}$ such that $f_{j}(x)>f_{j}\left(x_{0}\right)$.

Definition 2.2. A functional $F: X \times X \times R^{n} \rightarrow R$ (where $X \subset R^{n}$ ) is sublinear in its third component if for all $(x, u) \in X \times X$,

$$
\begin{gathered}
F\left(x, u ; a_{1}+a_{2}\right) \leqq F\left(x, u ; a_{1}\right)+F\left(x, u ; a_{2}\right) \text { for all } a_{1}, a_{2} \in R^{n} ; \\
F(x, u ; \alpha a)=\alpha F(x, u ; a) \text { for all } \alpha \in R_{+} \text {and for all } a \in R^{n} .
\end{gathered}
$$

For convenience, we write $F_{x, u}(a)=F(x, u, a)$.

We now introduce higher-order $(F, \alpha, \rho, d)$-convex function. Where, $F: X \times X \times R^{n} \rightarrow$ $R$ is a sublinear functional, $\alpha: X \times X \rightarrow R_{+} \backslash\{0\}, \rho \in R$ and $d: X \times X \rightarrow R$. Let $\Phi: X \rightarrow R$ and $h: X \times R^{n} \rightarrow R$ be differentiable real valued functions.

Definition 2.3. $\Phi$ is said to be higher-order $(F, \alpha, \rho, d)$-convex at $u \in X$ with respect to $h$ if, $\forall(x, p) \in X \times R^{n}$,

$$
\Phi(x)-\Phi(u) \geqq F_{x, u}\left(\alpha\left(\nabla_{x} \Phi(u)+\nabla_{p} h(u, p)\right)\right)+h(u, p)-p^{T} \nabla_{p} h(u, p)+\rho d^{2}(x, u) .
$$

Remark 2.1. (1) When $\alpha=1$, and $\rho=0$ or $d=0$, the higher-order $(F, \alpha, \rho, d)$-convexity reduces to higher-order $F$-convexity in [14].

(2) When $\alpha=1, \rho=0$ or $d=0$, and $h(u, p)=\frac{1}{2} p^{T} \nabla_{x x} \Phi(u) p$, the higher-order $(F, \alpha$, $\rho, d)$-convexity reduces to second order F-convexity in [15].

we now give an example of higher-order $(F, \alpha, \rho, d)$-convex function with respect to $h(u, p)$, which is not higher-order $F$-convex and second order $F$-convex.

Example 2.1. Let $X \subset R, X=\{x: x \geqq 1\}, f: X \rightarrow R, F: X \times X \times R \rightarrow R, h: X \times R \rightarrow R$ and $d: X \times X \rightarrow R$ given as follows

$$
f(x)=x+\frac{2}{x+1}, F_{x, u}(a)=|a|(x-u)^{2}, h(u, p)=\frac{p}{u+1}, d(x, u)=x-u .
$$

And let $u=1, \rho=-1, \alpha=\frac{3}{4}$. Then for all $(x, p) \in X \times R$

$$
\begin{aligned}
f(x)-f(u)= & \frac{x^{2}-x}{x+1} \geqq F_{x, u}\left(\frac{3}{4}\left(\nabla_{x} f(u)+\nabla_{p} h(u, p)\right)\right) \\
& +h(u, p)-p^{T} \nabla_{p} h(u, p)-d^{2}(x, u)=-\frac{1}{4}(x-1)^{2} .
\end{aligned}
$$

This implies $f(x)$ is a higher-order $(F, \alpha, \rho, d)$-convex function with respect to $h$ at $u$. But when we let $x=2, p=3$ and $x=6, p=3$ respectively, we have

$$
\begin{gathered}
f(2)-f(1)=\frac{2}{3}<F_{x, u}\left(\nabla_{x} f(u)+\nabla_{p} h(u, p)\right)+h(u, p)-p^{T} \nabla_{p} h(u, p)=\frac{3}{4}, \\
f(6)-f(1)=\frac{30}{7}<F_{x, u}\left(\nabla_{x} f(u)+\nabla_{x x} f(u)\right)-\frac{1}{2} p^{T} \nabla_{x x} f(u) p=\frac{66}{4} .
\end{gathered}
$$

Hence, $f$ is neither a higher-order $F$-convex function nor a second order $F$-convex function. From now on, suppose that the sublinear functional $F$ satisfies the following condition:

$$
F_{x, y}(a)+a^{T} y \geqq 0, \quad \forall a \in R_{+}^{n} .
$$




\section{Higher-order symmetric duality}

In the section, we consider the following multiobjective fractional symmetric dual problems: (MFP) Minimize $L(x, y, p)=\left(L_{1}\left(x, y, p_{1}\right), \ldots, L_{k}\left(x, y, p_{k}\right)\right)^{T}$ subject to

$$
\begin{aligned}
& \sum_{i=1}^{k} \lambda_{i}\left[\left(\nabla_{y} f_{i}(x, y)-z_{i}+\nabla_{p_{i}} H_{i}\left(x, y, p_{i}\right)\right)\right. \\
& \left.\quad-L_{i}\left(x, y, p_{i}\right)\left(\nabla_{y} g_{i}(x, y)+r_{i}+\nabla_{p_{i}} G_{i}\left(x, y, p_{i}\right)\right)\right] \leqq 0, \\
& y^{T} \sum_{i=1}^{k} \lambda_{i}\left[\left(\nabla_{y} f_{i}(x, y)-z_{i}+\nabla_{p_{i}} H_{i}\left(x, y, p_{i}\right)\right)\right. \\
& \left.\quad-L_{i}\left(x, y, p_{i}\right)\left(\nabla_{y} g_{i}(x, y)+r_{i}+\nabla_{p_{i}} G_{i}\left(x, y, p_{i}\right)\right)\right] \geqq 0, \\
& \lambda>0, \quad \lambda^{T} e=1, \quad z_{i} \in D_{i}, \quad r_{i} \in F_{i}, \quad i=1 \ldots, k .
\end{aligned}
$$

(MFD) Maximize $M(u, v, q)=\left(M_{1}\left(u, v, q_{1}\right), \ldots, M_{k}\left(u, v, q_{k}\right)\right)^{T}$ subject to

$$
\begin{aligned}
& \sum_{i=1}^{k} \lambda_{i}\left[\left(\nabla_{x} f_{i}(u, v)+w_{i}+\nabla_{q_{i}} \Phi_{i}\left(u, v, q_{i}\right)\right)\right. \\
& \left.\quad-M_{i}\left(u, v, q_{i}\right)\left(\nabla_{x} g_{i}(u, v)-t_{i}+\nabla_{q_{i}} \Psi_{i}\left(u, v, q_{i}\right)\right)\right] \geqq 0, \\
& u^{T} \sum_{i=1}^{k} \lambda_{i}\left[\left(\nabla_{x} f_{i}(u, v)+w_{i}+\nabla_{q_{i}} \Phi_{i}\left(u, v, q_{i}\right)\right)\right. \\
& \left.\quad-M_{i}\left(u, v, q_{i}\right)\left(\nabla_{x} g_{i}(u, v)-t_{i}+\nabla_{q_{i}} \Psi_{i}\left(u, v, q_{i}\right)\right)\right] \leqq 0, \\
& \lambda>0, \quad \lambda^{T} e=1, \quad w_{i} \in C_{i}, \quad t_{i} \in E_{i}, \quad i=1 \ldots, k .
\end{aligned}
$$

where

$$
\begin{aligned}
L_{i}\left(x, y, p_{i}\right) & =\frac{f_{i}(x, y)+s\left(x \mid C_{i}\right)-y^{T} z_{i}+H_{i}\left(x, y, p_{i}\right)-p_{i}^{T} \nabla_{p_{i}} H_{i}\left(x, y, p_{i}\right)}{g_{i}(x, y)-s\left(x \mid E_{i}\right)+\gamma^{T} r_{i}+G_{i}\left(x, y, p_{i}\right)-p_{i}^{T} \nabla_{p_{i}} G_{i}\left(x, y, p_{i}\right)}, \\
M_{i}\left(u, v, q_{i}\right) & =\frac{f_{i}(u, v)-s\left(v \mid D_{i}\right)+u^{T} w_{i}+\Phi_{i}\left(u, v, q_{i}\right)-q_{i}^{T} \nabla_{q_{i}} \Phi_{i}\left(u, v, q_{i}\right)}{g_{i}(u, v)+s\left(v \mid F_{i}\right)-u^{T} t_{i}+\Psi_{i}\left(u, v, q_{i}\right)-q_{i}^{T} \nabla_{q_{i}} \Psi_{i}\left(u, v, q_{i}\right)},
\end{aligned}
$$

$f_{i}: R_{n} \times R_{m} \rightarrow R ; g_{i}: R^{n} \times R^{m} \rightarrow R ; H_{i}, G_{i}: R^{n} \times R^{m} \rightarrow R$ and $\Phi_{i}, \Psi_{i}: R_{n} \times R_{m} \times R_{n} \rightarrow$ $R$ are twice differentiable functions for all $i=1 \ldots, k . C_{i}, E_{i}$ are compact convex sets in $R^{n}$, and $D_{i}, F_{i}$ are compact convex sets in $R^{m}, i=1, \ldots, k . e=(1, \ldots, 1)^{T} \in R^{k} . p_{i} \in R^{m}$, $q_{i} \in R^{n}, i=1, \ldots, k, p=\left(p_{1}, \ldots, p_{k}\right), q=\left(q_{1}, \ldots, q_{k}\right)$. It is assumed that in the feasible regions the numerators are nonnegative and denominators are positive.

We let $S=\left(S_{1}, \ldots, S_{k}\right)^{T}, W=\left(W_{1}, \ldots, W_{k}\right)^{T} \in R^{k}$. Then we can express the programs (MFP) and (MFD) equivalently as:

$(\mathbf{M F P})_{\mathbf{S}}$ Minimize $S$ subject to

$$
\begin{aligned}
& \left(f_{i}(x, y)+s\left(x \mid C_{i}\right)-y^{T} z_{i}+H_{i}\left(x, y, p_{i}\right)-p_{i}^{T} \nabla_{p_{i}} H_{i}\left(x, y, p_{i}\right)\right) \\
& \quad-S_{i}\left(g_{i}(x, y)-s\left(x \mid E_{i}\right)+y^{T} r_{i}+G_{i}\left(x, y, p_{i}\right)-p_{i}^{T} \nabla_{p_{i}} G_{i}\left(x, y, p_{i}\right)\right)=0, i=1, \ldots, k, \\
& \sum_{i=1}^{k} \lambda_{i}\left[\left(\nabla_{y} f_{i}(x, y)-z_{i}+\nabla_{p_{i}} H_{i}\left(x, y, p_{i}\right)\right)\right. \\
& \left.\quad-S_{i}\left(\nabla_{y} g_{i}(x, y)+r_{i}+\nabla_{p_{i}} G_{i}\left(x, y, p_{i}\right)\right)\right] \leqq 0,
\end{aligned}
$$




$$
\begin{aligned}
& y^{T} \sum_{i=1}^{k} \lambda_{i}\left[\left(\nabla_{y} f_{i}(x, y)-z_{i}+\nabla_{p_{i}} H_{i}\left(x, y, p_{i}\right)\right)\right. \\
& \left.\quad-S_{i}\left(\nabla_{y} g_{i}(x, y)+r_{i}+\nabla_{p_{i}} G_{i}\left(x, y, p_{i}\right)\right)\right] \geqq 0, \\
& \lambda>0, \quad \lambda^{T} e=1, \quad z_{i} \in D_{i}, \quad r_{i} \in F_{i}, \quad i=1 \ldots, k .
\end{aligned}
$$

$(\mathbf{M F D})_{\mathbf{W}}$ Maximize $W$ subject to

$$
\begin{aligned}
& \left(f_{i}(u, v)-s\left(v \mid D_{i}\right)+u^{T} w_{i}+\Phi_{i}\left(u, v, q_{i}\right)-q_{i}^{T} \nabla_{q_{i}} \Phi_{i}\left(u, v, q_{i}\right)\right) \\
& \quad-W_{i}\left(g_{i}(u, v)+s\left(v \mid F_{i}\right)-u^{T} t_{i}+\Psi_{i}\left(u, v, q_{i}\right)-q_{i}^{T} \nabla_{q_{i}} \Psi_{i}\left(u, v, q_{i}\right)\right)=0, \quad i=1, \ldots, k, \\
& \sum_{i=1}^{k} \lambda_{i}\left[\left(\nabla_{x} f_{i}(u, v)+w_{i}+\nabla_{q_{i}} \Phi_{i}\left(u, v, q_{i}\right)\right)\right. \\
& \left.\quad-W_{i}\left(\nabla_{x} g_{i}(u, v)-t_{i}+\nabla_{q_{i}} \Psi_{i}\left(u, v, q_{i}\right)\right)\right] \geqq 0, \\
& u^{T} \sum_{i=1}^{k} \lambda_{i}\left[\left(\nabla_{x} f_{i}(u, v)+w_{i}+\nabla_{q_{i}} \Phi_{i}\left(u, v, q_{i}\right)\right)\right. \\
& \left.\quad-W_{i}\left(\nabla_{x} g_{i}(u, v)-t_{i}+\nabla_{q_{i}} \Psi_{i}\left(u, v, q_{i}\right)\right)\right] \leqq 0, \\
& \lambda>0, \quad \lambda^{T} e=1, \quad w_{i} \in C_{i}, \quad t_{i} \in E_{i}, \quad i=1 \ldots, k .
\end{aligned}
$$

Now we can prove weak, strong and converse duality theorems for $(\mathrm{MFP})_{S}$ and $(\mathrm{MFD})_{W}$, but equally apply to (MFP) and (MFD).

Theorem 3.1 (Weak duality). Let $\left(x, y, S, z_{1}, \ldots, z_{k}, r_{1}, \ldots, r_{k}, \lambda, p\right)$ be feasible for $(\mathrm{MFD})_{S}$ and let $\left(u, v, W, w_{1}, \ldots, w_{k}, t_{1} \ldots, t_{k}, \lambda, q\right)$ be feasible for (MFD) $)_{W}$. Let $\forall i \in\{1$, $\ldots, k\}, f_{i}(., v)+(.)^{T} w_{i}$ be higher-order $\left(F, \alpha, \rho_{i}, d_{i}\right)$-convex at $u$ with respect to $\Phi_{i}(u, v$, $\left.q_{i}\right)$, - $\left(g_{i}(., v)-(.)^{T} t_{i}\right)$ be higher-order $\left(F, \alpha, \rho, d_{i}\right)$-convex at $u$ with respect to $-\Psi_{i}(u, v$, $\left.q_{i}\right),-\left(f_{i}(x,)-.(.)^{T} z_{i}\right)$ be higher-order $\left(K, \bar{\alpha}, \bar{\rho}_{i}, \bar{d}_{i}\right)$-convex at $y$ with respect to $-H_{i}(x, y$, $\left.p_{i}\right), g_{i}(x,)+.(.)^{T} r_{i}$ be higher-order $\left(K, \bar{\alpha}, \bar{\rho}_{i}, \bar{d}_{i}\right)$-convex at $y$ with respect to $G_{i}\left(x, y, p_{i}\right)$, where sublinear functional $F: R^{n} \times R^{n} \times R^{n} \rightarrow R$ and $K: R^{m} \times R^{m} \times R^{m} \rightarrow R$ satisfy the condition (1). If the following conditions hold:

$$
\begin{aligned}
& g_{i}(x, v)+v^{T} r_{i}-s\left(x \mid E_{i}\right)>0, i=1, \ldots, k \\
& \sum_{i=1}^{k} \lambda_{i}\left(\left(1+W_{i}\right) \rho_{i} d_{i}^{2}(x, u)+\left(1+S_{i}\right) \bar{\rho}_{i} \bar{d}_{i}^{2}(v, y)\right) \geqq 0 .
\end{aligned}
$$

\section{Then $S \$ W$.}

Proof. Since $\left(u, v, W, w_{1}, \ldots, w_{k}, t_{1} \ldots, t_{k}, \lambda, q\right)$ is feasible for (MFD) $)_{W}$, from (6), (7) and $F$ satisfies condition (1), it follows that

$$
F_{x, u}\left(\sum_{i=1}^{k} \lambda_{i}\left[\left(\nabla_{x} f_{i}(u, v)+w_{i}+\nabla_{q_{i}} \Phi_{i}\left(u, v, q_{i}\right)\right)-W_{i}\left(\nabla_{x} g_{i}(u, v)-t_{i}+\nabla_{q_{i}} \Psi_{i}\left(u, v, q_{i}\right)\right)\right]\right) \geqq 0 .
$$

Using the convexity assumptions of $f_{i}(., v)+(.)^{T} w_{i}$ and $-\left(g_{i}(., v)-(.)^{T} t_{i}\right)$ at $u$, we have 


$$
\begin{aligned}
& f_{i}(x, v)+x^{T} w_{i}-f_{i}(u, v)-u^{T} w_{i} \\
& \quad \geqq F_{x, u}\left(\alpha\left(\nabla_{x} f_{i}(u, v)+w_{i}+\nabla_{q_{i}} \Phi_{i}\left(u, v, q_{i}\right)\right)\right)+\Phi_{i}\left(u, v, q_{i}\right)-q_{i}^{T} \nabla_{q_{i}} \Phi_{i}\left(u, v, q_{i}\right)+\rho_{i} d_{i}^{2}(x, u), \\
& -g_{i}(x, v)+x^{T} t_{i}+g_{i}(u, v)-u^{T} t_{i} \\
& \quad \geqq F_{x, u}\left(\alpha\left(-\nabla_{x} g_{i}(u, v)+t_{i}-\nabla_{q_{i}} \Psi_{i}\left(u, v, q_{i}\right)\right)\right)-\Psi_{i}\left(u, v, q_{i}\right)+q_{i}^{T} \nabla_{q_{i}} \Phi_{i}\left(u, v, q_{i}\right)+\rho_{i} d_{i}^{2}(x, u) .
\end{aligned}
$$

Since $F$ is a sublinear functional and $\lambda>0, W \geqq 0, \alpha>0$, from (10) and the above two inequalities, we have

$$
\begin{aligned}
& \sum_{i=1}^{k} \lambda_{i}\left(f_{i}(x, v)+x^{T} w_{i}-f_{i}(u, v)-u^{T} w_{i}-\Phi_{i}\left(u, v, q_{i}\right)+q_{i}^{T} \nabla_{q_{i}} \Phi_{i}\left(u, v, q_{i}\right)\right) \\
& \quad+\sum_{i=1}^{k} \lambda_{i} W_{i}\left(g_{i}(u, v)+v^{T} r_{i}-u^{T} t_{i}+\Psi_{i}\left(u, v, q_{i}\right)-q_{i}^{T} \nabla_{q_{i}} \Psi_{i}\left(u, v, q_{i}\right)\right) \\
& \quad+\sum_{i=1}^{k} \lambda_{i} W_{i}\left(x^{T} t_{i}-g_{i}(x, v)-v^{T} r_{i}\right) \geqq \sum_{i=1}^{k} \lambda_{i}\left(1+W_{i}\right) \rho_{i} d_{i}^{2}(x, u) .
\end{aligned}
$$

Since $v^{T} r_{i} \leqq s\left(v \mid F_{i}\right)$, from (5) and (11), we have

$$
\sum_{i=1}^{k} \lambda_{i}\left[\left(f_{i}(x, v)+x^{T} w_{i}-s\left(v \mid D_{i}\right)\right)+W_{i}\left(x^{T} t_{i}-v^{T} r_{i}-g_{i}(x, v)\right)\right] \geqq \sum_{i=1}^{k} \lambda_{i}\left(1+W_{i}\right) \rho_{i} d_{i}^{2}(x, u) .
$$

On the other hand, from (3), (4) and sublinear functional $K$ satisfies condition (1), we obtain

$$
\begin{aligned}
& K_{v, y}\left(-\sum_{i=1}^{k} \lambda_{i}\left(\left(\nabla_{y} f_{i}(x, y)-z_{i}+\nabla_{p_{i}} H_{i}\left(x, y, p_{i}\right)\right)\right.\right. \\
& \left.\left.\quad-S_{i}\left(\nabla_{y} g_{i}(x, y)+r_{i}+\nabla_{p_{i}} G_{i}\left(x, y, p_{i}\right)\right)\right)\right) \geqq 0 .
\end{aligned}
$$

Using the convexity assumptions of $-f_{i}(x,)+.(.)^{T} z_{i}$ and $g_{i}(x,)+.(.)^{T} r_{i}$ at $y$, we have

$$
\begin{aligned}
-f_{i}(x, v)+v^{T} z_{i}+f_{i}(x, y)-y^{T} z_{i} \geqq & K_{v, y}\left(\bar{\alpha}\left(-\nabla_{y} f_{i}(x, y)+z_{i}-\nabla_{p_{i}} H_{i}\left(x, y, p_{i}\right)\right)\right) \\
& -H_{i}\left(x, y, p_{i}\right)+p_{i}^{T} \nabla_{p_{i}} H_{i}\left(x, y, p_{i}\right)+\bar{\rho}_{i} \bar{d}_{i}^{2}(v, y), \\
g_{i}(x, v)+v^{T} r_{i}-g_{i}(x, y)-y^{T} r_{i} \geqq & K_{v, y}\left(\bar{\alpha}\left(\nabla_{y} g_{i}(x, y)+r_{i}+\nabla_{p_{i}} G_{i}\left(x, y, p_{i}\right)\right)\right) \\
& +G_{i}\left(x, y, p_{i}\right)-p_{i}^{T} \nabla_{p_{i}} G_{i}\left(x, y, p_{i}\right)+\bar{\rho}_{i} \bar{d}_{i}^{2}(v, y) .
\end{aligned}
$$

Since $K$ is a sublinear functional, and $\lambda>0, S \geqq 0, \bar{\alpha}>0$, from (13) and the above two inequalities, it holds

$$
\begin{aligned}
& \sum_{i=1}^{k} \lambda_{i}\left(-f_{i}(x, v)+v^{T} z_{i}+f_{i}(x, y)-y^{T} z_{i}+H_{i}\left(x, y, p_{i}\right)-p_{i}^{T} \nabla_{p_{i}} H_{i}\left(x, y, p_{i}\right)\right) \\
& \quad+\sum_{i=1}^{k} \lambda_{i} S_{i}\left(-g_{i}(x, y)+x^{T} t_{i}-y^{T} r_{i}-G_{i}\left(x, y, p_{i}\right)+p_{i}^{T} \nabla_{p_{i}} G_{i}\left(x, y, p_{i}\right)\right) \\
& \quad+\sum_{i=1}^{k} \lambda_{i} S_{i}\left(g_{i}(x, v)+v^{T} r_{i}-x^{T} t_{i}\right) \geqq \sum_{i=1}^{k} \lambda_{i}\left(1+S_{i}\right) \bar{\rho}_{i} \bar{d}_{i}^{2}(v, y) .
\end{aligned}
$$


Since $x^{T} t_{i} \leqq s\left(x \mid E_{i}\right)$, from (2) and (14) we have

$$
\sum_{i=1}^{k} \lambda_{i}\left[\left(-f_{i}(x, v)+v^{T} z_{i}-s\left(x \mid C_{i}\right)\right)+S_{i}\left(g_{i}(x, v)+v^{T} r_{i}-x^{T} t_{i}\right)\right] \geqq \sum_{i=1}^{k} \lambda_{i}\left(1+S_{i}\right) \bar{\rho}_{i} \bar{d}_{i}^{2}(v, y) .
$$

Adding the above inequality and (12), we get

$$
\begin{aligned}
& \sum_{i=1}^{k} \lambda_{i}\left(v^{T} z_{i}-s\left(v \mid D_{i}\right)+x^{T} w_{i}-s\left(x \mid C_{i}\right)\right)+\sum_{i=1}^{k} \lambda_{i}\left(S_{i}-W_{i}\right)\left(g_{i}(x, v)+v^{T} r_{i}-x^{T} t_{i}\right) \\
& \quad \geqq \sum_{i=1}^{k} \lambda_{i}\left(\rho_{i} d_{i}^{2}(x, u)\left(1+W_{i}\right)+\bar{\rho}_{i} \bar{d}_{i}^{2}(v, y)\left(1+S_{i}\right)\right) .
\end{aligned}
$$

Since $\lambda_{i}>0, v^{T} z_{i}-s\left(v \mid D_{i}\right)+x^{T} w_{i}-s\left(x \mid C_{i}\right) \leqq 0, i=1, \ldots, k$, by (9) it yields

$$
\sum_{i=1}^{k} \lambda_{i}\left(S_{i}-W_{i}\right)\left(g_{i}(x, v)+v^{T} r_{i}-x^{T} t_{i}\right) \geqq 0 .
$$

By assumptions (8), we have $g_{i}(x, v)+v^{T} r_{i}-x^{T} t_{i}>0, i=1, \ldots, k$. Since $\lambda>0$, it follows that $S \$ W$. $\square$

Theorem 3.2 (Strong duality). Let $\left(\bar{x}, \bar{y}, \bar{S}, \bar{z}_{1}, \ldots, \bar{z}_{k}, \bar{r}_{1}, \ldots, \bar{r}_{k}, \bar{\lambda}, \bar{p}\right)$ be a properly efficient solution of $(\mathrm{MFP})_{S}$, and fix $\lambda=\bar{\lambda}$ in $(\mathrm{MFD})_{W}$. Suppose that $\nabla_{x} H_{i}(\bar{x}, \bar{y}, 0)=\nabla_{x} G_{i}(\bar{x}, \bar{y}, 0)=0, \nabla_{q_{i}} \Phi_{i}(\bar{x}, \bar{y}, 0)=\nabla_{q_{i}} \Psi_{i}(\bar{x}, \bar{y}, 0)=0$,

(a) $H_{i}(\bar{x}, \bar{y}, 0)=G_{i}(\bar{x}, \bar{y}, 0)=0, \Phi_{i}(\bar{x}, \bar{y}, 0)=\Psi_{i}(\bar{x}, \bar{y}, 0)=0, \nabla_{y} H_{i}(\bar{x}, \bar{y}, 0)=\nabla_{\gamma} G_{i}(\bar{x}, \bar{y}, 0)=0$, $\nabla_{p_{i}} H_{i}(\bar{x}, \bar{y}, 0)=\nabla_{p_{i}} G_{i}(\bar{x}, \bar{y}, 0)=0, i=1, \ldots, k$.

(b) For all $i \in\{1, \ldots, k\}$,

$$
f_{i}(\bar{x}, \bar{y})+s\left(\bar{x} \mid C_{i}\right)-\bar{y}^{T} \bar{z}_{i}+H_{i}\left(\bar{x}, \bar{y}, \bar{p}_{i}\right)-\bar{p}_{i}^{T} \nabla_{p_{i}} H_{i}\left(\bar{x}, \bar{y}, \bar{p}_{i}\right)>0 .
$$

(c) (i) $\nabla_{p_{i} p_{i}} H_{i}\left(\bar{x}, \bar{y}, \bar{p}_{i}\right)-\bar{S}_{i} \nabla_{p_{i} p_{i}} G_{i}\left(\bar{x}, \bar{y}, \bar{p}_{i}\right) \neq 0 \quad$ for $\quad \bar{p}_{i}=0, \quad i=1, \quad \ldots, \quad k$ and $\nabla_{p_{i} p_{i}} H_{i}\left(\bar{x}, \bar{y}, \bar{p}_{i}\right)-\bar{S}_{i} \nabla_{p_{i} p_{i}} G_{i}\left(\bar{x}, \bar{y}, \bar{p}_{i}\right)$ is nonsingular for all $i=1, \ldots, k$,

(ii) $\quad \sum_{i=1}^{k} \bar{\lambda}_{i}\left(\nabla_{\gamma \gamma} f_{i}(\bar{x}, \bar{y})-\bar{S}_{i} \nabla_{\gamma \gamma} g_{i}(\bar{x}, \bar{y})\right) \quad$ is positive definite and $\bar{p}_{i}^{T}\left(\left(\nabla_{\gamma} H_{i}\left(\bar{x}, \bar{y}, \bar{p}_{i}\right)-\bar{S}_{i} \nabla_{y} G_{i}\left(\bar{x}, \bar{y}, \bar{p}_{i}\right)\right)-\left(\nabla_{p_{i}} H_{i}\left(\bar{x}, \bar{y}, \bar{p}_{i}\right)-\bar{S}_{i} \nabla_{p_{i}} G_{i}\left(\bar{x}, \bar{y}, \bar{p}_{i}\right)\right)\right) \geqq 0$ for all $i=$ $1, \quad \ldots, \quad k$, or $\quad \sum_{i=1}^{k} \bar{\lambda}_{i}\left(\nabla_{y \gamma} f_{i}(\bar{x}, \bar{y})-\bar{S}_{i} \nabla_{y y} g_{i}(\bar{x}, \bar{y})\right) \quad$ is negative definite and $\bar{p}_{i}^{T}\left(\left(\nabla_{y} H_{i}\left(\bar{x}, \bar{y}, \bar{p}_{i}\right)-\bar{S}_{i} \nabla_{\gamma} G_{i}\left(\bar{x}, \bar{y}, \bar{p}_{i}\right)\right)-\left(\nabla_{p_{i}} H_{i}\left(\bar{x}, \bar{y}, \bar{p}_{i}\right)-\bar{S}_{i} \nabla_{p_{i}} G_{i}\left(\bar{x}, \bar{y}, \bar{p}_{i}\right)\right)\right) \leqq 0$ for all $i=$ $1, \ldots, k$.

(iii) $\left\{\nabla_{y} f_{i}(\bar{x}, \bar{y})-\bar{z}_{i}+\nabla_{p_{i}} H_{i}\left(\bar{x}, \bar{y}, \bar{p}_{i}\right)-\bar{S}_{i}\left(\nabla_{y} g_{i}(\bar{x}, \bar{y})+\bar{r}_{i}+\nabla_{p_{i}} G_{i}\left(\bar{x}, \bar{y}, \bar{p}_{i}\right)\right): i=1, \ldots, k\right\} \quad$ is linearly independent.

Then $\bar{p}=0$, and there exist $\bar{w}_{i} \in C_{i}$ and $\bar{t}_{i} \in E_{i}, i=1, \ldots, k$ such that $\left(\bar{x}, \bar{y}, \bar{S}, \bar{w}_{1}, \ldots, \bar{w}_{k}, \bar{t}_{1}, \ldots, \bar{t}_{k}, \bar{\lambda}, \bar{q}=0\right)$ is a feasible solution of (MFD) $)_{W}$. Furthermore, if the hypotheses in Theorem 3.1 are satisfied, then $\left(\bar{x}, \bar{y}, \bar{S}, \bar{w}_{1}, \ldots, \bar{w}_{k}, \bar{t}_{1}, \ldots, \bar{t}_{k}, \bar{\lambda}, \bar{q}=0\right)$ is a properly efficient solution of (MFD) $)_{W}$, and the two objective values are equal.

Proof. Since $\left(\bar{x}, \bar{y}, \bar{S}_{,} \bar{z}_{1}, \ldots, \bar{z}_{k}, \bar{r}_{1}, \ldots, \bar{r}_{k}, \bar{\lambda}, \bar{p}\right)$ is a properly efficient solution of $(\mathrm{MFP})_{S}$, by the Fritz John type necessary optimality conditions [16], there exist $\alpha \in R^{k}$, $\beta \in R^{k}, \gamma \in R^{m}, \delta \in R, \mu \in R^{k}$ and $\bar{w}_{i} \in R^{n}, \bar{t}_{i} \in R^{n}, i=1, \ldots, k$ such that 


$$
\begin{aligned}
& \sum_{i=1}^{k} \beta_{i}\left(\left(\nabla_{x} f_{i}(\bar{x}, \bar{y})+\bar{w}_{i}+\nabla_{x} H_{i}\left(\bar{x}, \bar{y}, \bar{p}_{i}\right)\right)-\bar{S}_{i}\left(\nabla_{x} g_{i}(\bar{x}, \bar{y})-\bar{t}_{i}+\nabla_{x} G_{i}\left(\bar{x}, \bar{y}, \bar{p}_{i}\right)\right)\right) \\
& +(\gamma-\delta \bar{y})^{T} \sum_{i=1}^{k} \bar{\lambda}_{i}\left(\nabla_{y x} f_{i}(\bar{x}, \bar{y})-\bar{S}_{i} \nabla_{\gamma x} g_{i}(\bar{x}, \bar{y})\right) \\
& +\sum_{i=1}^{k}\left(\nabla_{p_{i x}} H_{i}\left(\bar{x}, \bar{y}, \bar{p}_{i}\right)-\bar{S}_{i} \nabla_{p_{i} x} G_{i}\left(\bar{x}, \bar{y}, \bar{p}_{i}\right)\right)^{T}\left((\gamma-\delta \bar{y}) \bar{\lambda}_{i}-\beta_{i} \bar{p}_{i}\right)=0, \\
& \sum_{i=1}^{k}\left(\beta_{i}-\delta \bar{\lambda}_{i}\right)\left(\left(\nabla_{y} f_{i}(\bar{x}, \bar{y})-z_{i}+\nabla_{p_{i}} H_{i}\left(\bar{x}, \bar{y}, \bar{p}_{i}\right)\right)-\bar{S}_{i}\left(\nabla_{y} g_{i}(\bar{x}, \bar{y})+\bar{r}_{i}+\nabla_{p_{i}} G_{i}\left(\bar{x}, \bar{y}, \bar{p}_{i}\right)\right)\right) \\
& +\sum_{i=1}^{k} \beta_{i}\left(\left(\nabla_{\gamma} H_{i}\left(\bar{x}, \bar{y}, \bar{p}_{i}\right)-\bar{S}_{i} \nabla_{\gamma} G_{i}\left(\bar{x}, \bar{y}, \bar{p}_{i}\right)\right)-\left(\nabla_{p_{i}} H_{i}\left(\bar{x}, \bar{y}, \bar{p}_{i}\right)-\bar{S}_{i} \nabla_{p_{i}} G_{i}\left(\bar{x}, \bar{y}, \bar{p}_{i}\right)\right)\right) \\
& +\sum_{i=1}^{k} \bar{\lambda}_{i}\left(\left(\nabla_{\gamma y} f_{i}(\bar{x}, \bar{y})-\bar{S}_{i} \nabla_{y y} g_{i}(\bar{x}, \bar{y})\right)^{T}(\gamma-\delta \bar{y})\right) \\
& +\sum_{i=1}^{k}\left(\nabla_{p_{i} \gamma} H_{i}\left(\bar{x}, \bar{y}, \bar{p}_{i}\right)-\bar{S}_{i} \nabla_{p_{i} \gamma} G_{i}\left(\bar{x}, \bar{y}, \bar{p}_{i}\right)\right)^{T}\left(-\beta_{i} \bar{p}_{i}+(\gamma-\delta \bar{y}) \bar{\lambda}_{i}\right)=0, \\
& \alpha_{i}-\beta_{i}\left(g_{i}(\bar{x}, \bar{y})-s\left(\bar{x} \mid E_{i}\right)+\bar{y}^{T} \bar{r}_{i}+G_{i}\left(\bar{x}, \bar{y}, \bar{p}_{i}\right)-\bar{p}_{i}^{T} \nabla_{p_{i}} G_{i}\left(\bar{x}, \bar{y}, \bar{p}_{i}\right)\right) \\
& -(\gamma-\delta \bar{y})^{T}\left(\bar{\lambda}_{i}\left(\nabla_{\gamma} g_{i}(\bar{x}, \bar{y})+\bar{r}_{i}+\nabla_{p_{i}} G_{i}\left(\bar{x}, \bar{y}, \bar{p}_{i}\right)\right)\right)=0, \quad i=1, \ldots, k, \\
& (\gamma-\delta \bar{y})^{T}\left(\left(\nabla_{\gamma} f_{i}(\bar{x}, \bar{y})-\bar{z}_{i}+\nabla_{p_{i}} H_{i}\left(\bar{x}, \bar{y}, \bar{p}_{i}\right)-\bar{S}_{i}\left(\nabla_{y} g_{i}(\bar{x}, \bar{y})+\bar{r}_{i}+\nabla_{p_{i}} G_{i}\left(\bar{x}, \bar{y}, \bar{p}_{i}\right)\right)\right)\right. \\
& -\mu_{i}=0, \quad i=1, \ldots, k, \\
& \left(\bar{\lambda}_{i}(\gamma-\delta \bar{y})-\beta_{i} \bar{p}_{i}\right)^{T}\left(\nabla_{p_{i} p_{i}} H_{i}\left(\bar{x}, \bar{y}, \bar{p}_{i}\right)-\bar{S}_{i} \nabla_{p_{i} p_{i}} G_{i}\left(\bar{x}, \bar{y}, \bar{p}_{i}\right)\right)=0, \quad i=1, \ldots, k, \\
& \beta_{i} \bar{y}+(\gamma-\delta \bar{y}) \bar{\lambda}_{i} \in N_{D_{i}}\left(\bar{z}_{i}\right), \quad i=1, \ldots, k, \\
& \beta_{i} \bar{S}_{i} \bar{y}+\bar{\lambda}_{i} \bar{S}_{i}(\gamma-\delta \bar{y}) \in N_{F_{i}}\left(\bar{r}_{i}\right), \quad i=1, \ldots, k \\
& \gamma^{T} \sum_{i=1}^{k} \bar{\lambda}_{i}\left(\left(\nabla_{\gamma} f_{i}(\bar{x}, \bar{y})-\bar{z}_{i}+\nabla_{p_{i}} H_{i}\left(\bar{x}, \bar{y}, \bar{p}_{i}\right)\right)\right. \\
& \left.-\bar{S}_{i}\left(\nabla_{\gamma} g_{i}(\bar{x}, \bar{y})+\bar{r}_{i}+\nabla_{p_{i}} G_{i}\left(\bar{x}, \bar{y}, \bar{p}_{i}\right)\right)\right)=0, \\
& \delta \bar{y}^{T} \sum_{i=1}^{k} \bar{\lambda}_{i}\left(\left(\nabla_{\gamma} f_{i}(\bar{x}, \bar{y})-\bar{z}_{i}+\nabla_{p_{i}} H_{i}\left(\bar{x}, \bar{y}, \bar{p}_{i}\right)\right)\right. \\
& \left.-\bar{S}_{i}\left(\nabla_{\gamma} g_{i}(\bar{x}, \bar{y})+\bar{r}_{i}+\nabla_{p_{i}} G_{i}\left(\bar{x}, \bar{y}, \bar{p}_{i}\right)\right)\right)=0, \\
& \mu^{T} \bar{\lambda}=0, \\
& \bar{w}_{i} \in C_{i}, \bar{t}_{i} \in E_{i}, \bar{x}^{T} \bar{t}_{i}=s\left(\bar{x} \mid E_{i}\right), \bar{x}^{T} \bar{w}_{i}=s\left(\bar{x} \mid C_{i}\right), \quad i=1, \ldots, k,
\end{aligned}
$$


$(\alpha, \beta, \gamma, \delta, \mu) \neq 0,(\alpha, \gamma, \delta, \mu) \geqq 0$.

Since $\bar{\lambda}>0$, and $\mu \geqq 0$, (24) implies $\mu=0$. Consequently, (18) yields

$$
\begin{aligned}
& (\gamma-\delta \bar{y})^{T}\left(\left(\nabla_{y} f_{i}(\bar{x}, \bar{y})-\bar{z}_{i}+\nabla_{p_{i}} H_{i}\left(\bar{x}, \bar{y}, \bar{p}_{i}\right)\right.\right. \\
& \left.\quad-\bar{S}_{i}\left(\nabla_{y} g_{i}(\bar{x}, \bar{y})+\bar{r}_{i}+\nabla_{p_{i}} G_{i}\left(\bar{x}, \bar{y}, \bar{p}_{i}\right)\right)\right)=0, i=1, \ldots, k .
\end{aligned}
$$

By assumption (i) and (19), we have

$$
\bar{\lambda}_{i}(\gamma-\delta \bar{y})=\beta_{i} \bar{p}_{i,} \quad i=1, \ldots, k .
$$

Multiplying (16) $(\gamma-\delta \bar{y})$ by left, from (27) and (28) we have

$$
\begin{aligned}
& (\gamma-\delta \bar{y})^{T} \sum_{i=1}^{k} \beta_{i}\left(\left(\nabla_{y} H_{i}\left(\bar{x}, \bar{y}, \bar{p}_{i}\right)-\bar{S}_{i} \nabla_{y} G_{i}\left(\bar{x}, \bar{y}, \bar{p}_{i}\right)\right)-\left(\nabla_{p_{i}} H_{i}\left(\bar{x}, \bar{y}, \bar{p}_{i}\right)-\bar{S}_{i} \nabla_{p_{i}} G_{i}\left(\bar{x}, \bar{y}, \bar{p}_{i}\right)\right)\right) \\
& \quad+(\gamma-\delta \bar{y})^{T} \sum_{i=1}^{k} \bar{\lambda}_{i}\left(\nabla_{\gamma y} f_{i}(\bar{x}, \bar{y})-\bar{S}_{i} \nabla_{y \gamma} g_{i}(\bar{x}, \bar{y})\right)(\gamma-\delta \bar{y})=0 .
\end{aligned}
$$

Since $\bar{\lambda}>0$, from (28) and the above equation, we have

$$
\begin{aligned}
& \sum_{i=1}^{k} \frac{\beta_{i}^{2}}{\bar{\lambda}_{i}} \bar{p}_{i}^{T}\left(\left(\nabla_{y} H_{i}\left(\bar{x}, \bar{y}, \bar{p}_{i}\right)-\bar{S}_{i} \nabla_{y} G_{i}\left(\bar{x}, \bar{y}, \bar{p}_{i}\right)\right)-\left(\nabla_{p_{i}} H_{i}\left(\bar{x}, \bar{y}, \bar{p}_{i}\right)-\bar{S}_{i} \nabla_{p_{i}} G_{i}\left(\bar{x}, \bar{y}, \bar{p}_{i}\right)\right)\right) \\
& \quad+(\gamma-\delta \bar{y})^{T} \sum_{i=1}^{k} \bar{\lambda}_{i}\left(\nabla_{\gamma y} f_{i}(\bar{x}, \bar{y})-\bar{S}_{i} \nabla_{y \gamma} g_{i}(\bar{x}, \bar{y})\right)(\gamma-\delta \bar{y})=0 .
\end{aligned}
$$

Which by assumption (ii), we can obtain

$$
\gamma-\delta \bar{y}=0 .
$$

Using (29) in (28), we have $\beta_{i} \bar{p}_{i}=0, i=1, \ldots, k$. This implies that $\bar{p}_{i}=0$ when $\beta_{i} \neq$ 0 , for all $i \in\{1, \ldots, k\}$. Hence, by assumption (1), we get

$$
\sum_{i=1}^{k} \beta_{i}\left(\left(\nabla_{\gamma} H_{i}\left(\bar{x}, \bar{y}, \bar{p}_{i}\right)-\bar{S}_{i} \nabla_{\gamma} G_{i}\left(\bar{x}, \bar{y}, \bar{p}_{i}\right)\right)-\left(\nabla_{p_{i}} H_{i}\left(\bar{x}, \bar{y}, \bar{p}_{i}\right)-\bar{S}_{i} \nabla_{p_{i}} G_{i}\left(\bar{x}, \bar{y}, \bar{p}_{i}\right)\right)\right)=0 .
$$

Combining this with (16), (28) and (29), it follows that

$$
\sum_{i=1}^{k}\left(\beta_{i}-\delta \bar{\lambda}_{i}\right)\left(\nabla_{y} f_{i}(\bar{x}, \bar{y})-\bar{z}_{i}+\nabla_{p_{i}} H_{i}\left(\bar{x}, \bar{y}, \bar{p}_{i}\right)-\bar{S}_{i}\left(\nabla_{y} g_{i}(\bar{x}, \bar{y})+\bar{r}_{i}+\nabla_{p_{i}} G_{i}\left(\bar{x}, \bar{y}, \bar{p}_{i}\right)\right)\right)=0,
$$

which by assumption (iii), it yields

$$
\beta_{i}-\delta \bar{\lambda}_{i}=0, \quad i=1, \ldots, k .
$$

We claim that $\delta \neq 0$, otherwise, from (29) and (30) we get $\beta=0, \gamma=0$. Using (29) in (17), we get $\alpha=0$. This contradicts with (26). Hence $\delta=0$. Since $\bar{\lambda}>0$, from (30) we get $\beta>0$. Hence $\beta_{i} \bar{p}_{i}=0, i=1, \ldots, k$ implies $\bar{p}_{i}=0, i=1, \ldots, k$. Using (28), (29) and the fact $\bar{p}_{i}=0, i=1, \ldots, k$ in (15), by assumption (a), we get

$$
\sum_{i=1}^{k} \beta_{i}\left(\left(\nabla_{x} f_{i}(\bar{x}, \bar{y})+\bar{w}_{i}\right)-\bar{S}_{i}\left(\nabla_{x} g_{i}(\bar{x}, \bar{y})-\bar{t}_{i}\right)\right)=0,
$$


combining this with (30) and $\delta>0, \bar{\lambda}>0$, it holds

$$
\sum_{i=1}^{k} \bar{\lambda}_{i}\left(\left(\nabla_{x} f_{i}(\bar{x}, \bar{y})+\bar{w}_{i}\right)-\bar{S}_{i}\left(\nabla_{x} g_{i}(\bar{x}, \bar{y})-\bar{t}_{i}\right)\right)=0,
$$

which yields

$$
\bar{x}^{T} \sum_{i=1}^{k} \bar{\lambda}_{i}\left(\left(\nabla_{x} f_{i}(\bar{x}, \bar{y})+\bar{w}_{i}\right)-\bar{S}_{i}\left(\nabla_{x} g_{i}(\bar{x}, \bar{y})-\bar{t}_{i}\right)\right)=0 .
$$

On the other hand, by assumption ( $a$ ) and (2) we get

$$
\left(f_{i}(\bar{x}, \bar{y})+s\left(\bar{x} \mid C_{i}\right)-\bar{y}^{T} \bar{z}_{i}\right)-\bar{S}_{i}\left(g_{i}(\bar{x}, \bar{y})-s\left(\bar{x} \mid E_{i}\right)+\bar{y}^{T} \bar{r}_{i}\right)=0, \quad i=1, \ldots, k .
$$

Since $\beta>0$, by (20) and (29) we get $\bar{y} \in N_{D_{i}}\left(\bar{z}_{i}\right), i=1, \ldots, k$. This implies

$$
\bar{y}^{T} \bar{z}_{i}=s\left(\bar{y} \mid D_{i}\right), \quad i=1, \ldots, k .
$$

Assumption (b) implies $\bar{S}>0$. By (21), we similarly have $\bar{y} \in N_{F_{i}}\left(\bar{r}_{i}\right), i=1, \ldots, k$. This implies

$$
\bar{y}^{T} \bar{r}_{i}=s\left(\bar{y} \mid F_{i}\right), \quad i=1, \ldots, k .
$$

Combining (25), (33), (34) and (35), we get

$$
\left(f_{i}(\bar{x}, \bar{y})+\bar{x}^{T} \bar{w}_{i}-s\left(\bar{y} \mid D_{i}\right)\right)-\bar{S}_{i}\left(g_{i}(\bar{x}, \bar{y})-\bar{x}^{T} \bar{t}_{i}+s\left(\bar{y} \mid F_{i}\right)=0, \quad i=1, \ldots, k,\right.
$$

combining this with (31) and (32), by assumption (a), $\left(\bar{x}, \bar{y}, \bar{S}, \bar{w}_{1}, \ldots, \bar{w}_{k}, \bar{t}_{1}, \ldots, \bar{t}_{k}, \bar{\lambda}, \bar{q}=0\right)$ is a feasible solution of (MFD) $W$.

Under the assumptions of Theorem 3.1 , if $\left(\bar{x}, \bar{y}, \bar{S}, \bar{w}_{1}, \ldots, \bar{w}_{k}, \bar{t}_{1}, \ldots, \bar{t}_{k}, \bar{\lambda}, \bar{q}=0\right)$ is not an efficient solution of $(M F D)_{W}$, then there exists other feasible solution $\left(u, v, W, w_{1}, \ldots, w_{k}, t_{1}, \ldots, t_{k}, \bar{\lambda}, q\right)$, of $(\mathrm{MFD})_{W}$ such that $\bar{S} \leq W$. Since $\left(\bar{x}, \bar{y}, \bar{S}, \bar{z}_{1}, \ldots, \bar{z}_{k}, \bar{r}_{1}, \ldots, \bar{r}_{k}, \bar{\lambda}, \bar{p}\right)$ is a feasible solution of $(\mathrm{MFP})_{S}$, by Theorem 3.1, we have $\bar{S} \not \leq W$, hence the contradiction implies $\left(\bar{x}, \bar{y}, \bar{S}, \bar{w}_{1}, \ldots, \bar{w}_{k}, \bar{t}_{1}, \ldots, \bar{t}_{k}, \bar{\lambda}, \bar{q}=0\right)$ is an efficient solution of (MFD) $)_{W}$

If $\left(\bar{x}, \bar{y}, \bar{S}, \bar{w}_{1}, \ldots, \bar{w}_{k}, \bar{t}_{1}, \ldots, \bar{t}_{k}, \bar{\lambda}, \bar{q}=0\right)$ is not a properly efficient solution of (MFD) $W$, then there exists other feasible solution $\left(u, v, W, w_{1}, \ldots, w_{k}, t_{1}, \ldots, t_{k}, \bar{\lambda}, q\right)$ of (MFD) $W$ such that for an index $i \in\{1, \ldots, k\}$ and any real number $M>0, W_{i}-\bar{S}_{i}>M\left(\bar{S}_{j}-W_{j}\right)$ for $j$ satisfying $\bar{S}_{j}>W_{j}$ whenever $W_{i}>\bar{S}_{i}$ This implies $W_{i}>\bar{S}_{i}$ can be made arbitrarily large and this contradicts with Theorem 3.1. And it is easy to find that the two objective values are equal.

Theorem 3.3 (Strict converse duality). Let $\left(\bar{u}, \bar{v}, \bar{W}, \bar{w}_{1}, \ldots, \bar{w}_{k}, \bar{t}_{1}, \ldots, \bar{t}_{k}, \bar{\lambda}, \bar{q}\right)$ be a properly efficient solution of $(\mathrm{MFD})_{W}$, and fix $\lambda=\bar{\lambda}$ in $(\mathrm{MFP})_{S}$. Suppose that

$$
\nabla_{x} \Phi_{i}(\bar{u}, \bar{v}, 0)=\nabla_{x} \Psi_{i}(\bar{u}, \bar{v}, 0)=0, \nabla_{q_{i}} \Phi_{i}(\bar{u}, \bar{v}, 0)=\nabla_{q_{i}} \Psi_{i}(\bar{u}, \bar{v}, 0)=0,
$$

(a) $H_{i}(\bar{u}, \bar{v}, 0)=G_{i}(\bar{u}, \bar{v}, 0)=0, \Phi_{i}(\bar{u}, \bar{v}, 0)=\Psi_{i}(\bar{u}, \bar{v}, 0)=0, \nabla_{\gamma} \Phi_{i}(\bar{u}, \bar{v}, 0)=\nabla_{\gamma} \Psi_{i}(\bar{u}, \bar{v}, 0)=0$, $\nabla_{p_{i}} H_{i}(\bar{u}, \bar{v}, 0)=\nabla_{p_{i}} G_{i}(\bar{u}, \bar{v}, 0)=0, \quad i=1, \ldots, k$.

(b) For all $i \in\{1, \ldots, k\}$,

$$
f_{i}(\bar{u}, \bar{v})-s\left(\bar{v} \mid D_{i}\right)+\bar{u}^{T} \bar{w}_{i}+\Phi_{i}\left(\bar{u}, \bar{v}, \bar{q}_{i}\right)-\bar{q}_{i}^{T} \nabla_{q_{i}} \Phi_{i}\left(\bar{u}, \bar{v}, \bar{q}_{i}\right)>0 .
$$


(c) (i) $\nabla_{q_{i} q_{i}} \Phi_{i}\left(\bar{u}, \bar{v}, \bar{q}_{i}\right)-\bar{W}_{i} \nabla_{q_{i} q_{i}} \Psi_{i}\left(\bar{u}, \bar{v}, \bar{q}_{i}\right) \neq 0$, for $\bar{q}_{i}=0, \quad i=1, \ldots, k$, and $\nabla_{q_{i} q_{i}} \Phi_{i}\left(\bar{u}, \bar{v}, \bar{q}_{i}\right)-\bar{W}_{i} \nabla_{q_{i} q_{i}} \Psi_{i}\left(\bar{u}, \bar{v}, \bar{q}_{i}\right)$ is nonsingular for all $i=1, \ldots, k$, and

(ii) $\quad \sum_{i=1}^{k} \bar{\lambda}_{i}\left(\nabla_{x x} f_{i}(\bar{u}, \bar{v})-\bar{W}_{i} \nabla_{x x} g_{i}(\bar{u}, \bar{v})\right) \quad$ is positive definite and $\bar{q}_{i}^{T}\left(\left(\nabla_{x} \Phi_{i}\left(\bar{u}, \bar{v}, \bar{q}_{i}\right)-\bar{W}_{i} \nabla_{x} \Psi_{i}\left(\bar{u}, \bar{v}, \bar{q}_{i}\right)\right)-\left(\nabla_{q_{i}} \Phi_{i}\left(\bar{u}, \bar{v}, \bar{q}_{i}\right)-\bar{W}_{i} \nabla_{q_{i}} \Psi_{i}\left(\bar{u}, \bar{v}, \bar{q}_{i}\right)\right)\right) \geqq 0$ for all $i=$ $1, \quad \ldots, \quad k, \quad$ or $\sum_{i=1}^{k} \bar{\lambda}_{i}\left(\nabla_{x x} f_{i}(\bar{u}, \bar{v})-\bar{W}_{i} \nabla_{x x} g_{i}(\bar{u}, \bar{v})\right) \quad$ is negative definite and $\bar{q}_{i}^{T}\left(\left(\nabla_{x} \Phi_{i}\left(\bar{u}, \bar{v}, \bar{q}_{i}\right)-\bar{W}_{i} \nabla_{x} \Psi_{i}\left(\bar{u}, \bar{v}, \bar{q}_{i}\right)\right)-\left(\nabla_{q_{i}} \Phi_{i}\left(\bar{u}, \bar{v}, \bar{q}_{i}\right)-\bar{W}_{i} \nabla_{q_{i}} \Psi_{i}\left(\bar{u}, \bar{v}, \bar{q}_{i}\right)\right)\right) \leqq 0$ for all $i=$ $1, \ldots, k$.

(iii) $\left\{\nabla_{x} f_{i}(\bar{u}, \bar{v})+\bar{w}_{i}+\nabla_{q_{i}} \Phi_{i}\left(\bar{u}, \bar{v}, \bar{q}_{i}\right)-\bar{W}_{i}\left(\nabla_{x} g_{i}(\bar{u}, \bar{v})-\bar{t}_{i}+\nabla_{q_{i}} \Psi_{i}\left(\bar{u}, \bar{v}, \bar{q}_{i}\right)\right): i=1, \ldots, k\right\} \quad$ is linearly independent.

Then $\bar{q}=0$, and there exist $\bar{z}_{i} \in D_{i}$ and $\bar{r}_{i} \in F_{i}, i=1, \ldots, k$ such that $\left(\bar{u}, \bar{v}, \bar{W}, \bar{z}_{1}, \ldots, \bar{z}_{k}, \bar{r}_{1}, \ldots, \bar{r}_{k}, \bar{\lambda}, \bar{p}=0\right)$ is a feasible solution of $(\text { MFP })_{S}$. Furthermore, if the hypotheses in Theorem 3.1 are satisfied, then $\left(\bar{u}, \bar{v}, \bar{W}, \bar{z}_{1}, \ldots, \bar{z}_{k}, \bar{r}_{1}, \ldots, \bar{r}_{k}, \bar{\lambda}, \bar{p}=0\right)$ is a properly efficient solution of $(\mathrm{MFP})_{S}$, and the two objective values are equal. $\square$

Remark 3.1.(1) If $k=1, \quad H_{1}\left(x, y, p_{1}\right)=\frac{1}{2} p_{1}^{T} \nabla_{\gamma \gamma} f_{1}(x, y) p_{1}$, $\Phi_{1}\left(u, v, q_{1}\right)=\frac{1}{2} q_{1}^{T} \nabla_{x x} f_{1}(u, v) q_{1}, \quad \Phi_{1}\left(u, v, q_{1}\right)=\frac{1}{2} q_{1}^{T} \nabla_{x x} f_{1}(u, v) q_{1}, \quad$ and $g_{1}(u, v)+s\left(v \mid F_{1}\right)-u^{T} t_{1}+\Psi_{1}\left(u, v, q_{1}\right)-q_{1}^{T} \nabla_{q 1} \Psi_{1}\left(u, v, q_{1}\right)=1$, then (MFP) $)_{S}$ and (MFD) $W$ becomes the problems considered by Hou and Yang [17].

(2) If $k=1, g_{1}(x, y)-s\left(x \mid E_{1}\right)+y^{T} r_{1}+G_{1}\left(x, y, p_{1}\right)-p_{1}^{T} \nabla_{p_{1}} G_{1}\left(x, y, p_{1}\right)=1$, and $g_{1}(u, v)+s\left(v \mid F_{1}\right)-u^{T} t_{1}+\Psi_{1}\left(u, v, q_{1}\right)-q_{1}^{T} \nabla_{q 1} \Psi_{1}\left(u, v, q_{1}\right)=1$, then (MFP) $)_{S}$ and (MFD) ${ }_{W}$ becomes the problems considered by Mishra [18].

$$
\text { If } \quad g_{i}(x, y)-s\left(x \mid E_{i}\right)+y^{T} r_{i}+G_{i}\left(x, y, p_{i}\right)-p_{i}^{T} \nabla_{p_{i}} G_{i}\left(x, y, p_{i}\right)=1, \quad \text { and }
$$
$g_{i}(u, v)+s\left(v \mid F_{i}\right)-u^{T} t_{i}+\Psi_{i}\left(u, v, q_{i}\right)-q_{i}^{T} \nabla_{q_{i}} \Psi_{i}\left(u, v, q_{i}\right)=1$ for all $i\{1, \ldots, k\}$, then $(\mathrm{MFP})_{S}$ and $(\mathrm{MFD})_{W}$ becomes the problems considered by Chen [14].

$$
\text { If } \quad g_{i}(x, y)-s\left(x \mid E_{i}\right)+y^{T} r_{i}+G_{i}\left(x, y, p_{i}\right)-p_{i}^{T} \nabla_{p_{i}} G_{i}\left(x, y, p_{i}\right)=1,
$$

$H_{i}\left(x, y, p_{i}\right)=\frac{1}{2} p_{i}^{T} \nabla_{\gamma \gamma} f_{i}(x, y) p_{i}, \Phi_{i}\left(u, v, q_{i}\right)=\frac{1}{2} q_{i}^{T} \nabla_{x x} f_{i}(u, v) q_{i}$,

$H_{i}\left(x, y, p_{i}\right)=\frac{1}{2} p_{i}^{T} \nabla_{y \gamma} f_{i}(x, y) p_{i}, \Phi_{i}\left(u, v, q_{i}\right)=\frac{1}{2} q_{i}^{T} \nabla_{x x} f_{i}(u, v) q_{i}$, for all $i \in\{1, \ldots, k\}$, and there is not the condition $\lambda^{T} e=1$ in $(\mathrm{MFP})_{S}$ and (MFD) $)_{W}$, then the two problems reduce to the problems considered by Yang et al. [19].

\section{Acknowledgements}

This work was partially supported by the National Science Foundation of China (11126348), the Education Committee Project Research Foundation of Chongqing (KJ110624, KJ120628), the special fund of Chongqing key laboratory (CSTC,2011KLORSE03) and the Doctoral Foundation of Chongqing Normal University(No.10XLB015).

\section{Competing interests}

The authors declare that they have no competing interests.

Received: 28 December 2011 Accepted: 20 June 2012 Published: 20 June 2012

\section{References}

Dorn, WS: A symmetric dual theorem for quadratic programs. J Oper Res Soc Jpn. 2, 93-97 (1960)

Dantzig, GB, Eisenberg, E, Cottle, RW: Symmetric dual nonlinear programs. Pacific J Math. 15, 809-812 (1965)

Mond, B: A symmetric dual theorem for nonlinear programs. Q J Appl Math. 23, 265-269 (1965) 
4. Mond, B, Weir, T: Generalized concavity and duality. In: Schaible S, Ziemba WT (eds.) Generalized Concavity in Optimization and Economics. Academic Press, New York (1981)

5. Mond, B, Weir, T: Symmetric duality for nonlinear multiobjective programming. In: Kumar S (ed.) Recent Developments in Mathematical Programming. pp. 137-153. Gordon and Breach Science, London (1991)

6. Weir, T, Mond, B: Symmetric and self duality in multiple objective programming. Asia Pacific J Oper Res. 4, 124-133 (1988)

7. Gulati, TR, Husain, I, Ahmed, A: Multiobjective symmetric duality with invexity. Bull Aust Math Soc. 56, 25-36 (1997). doi:10.1017/S0004972700030707

8. Chandra, S, Craven, BD, Mond, B: Symmetric dual fractional programming. Z Oper Res. 29, $59-64$ (1985)

9. Pandey, S: Duality for multiobjective fractional programming involving generalized $\eta$-bonvex functions. Opsearch. 28 , 36-43 (1991)

10. Yang, XM, Wang, SY, Dneg, XT: Symmetric duality for a class of multiobjective fractional programming problems. J Math Anal Appl. 274, 279-295 (2002). doi:10.1016/50022-247X(02)00299-8

11. Mangasarian, OL: Second-and higher-order duality in nonlinear programming. J Math Anal Appl. 51, 607-620 (1975). doi:10.1016/0022-247X(75)90111-0

12. Mond, B, Zhang, J: Higher-order invexity and duality in mathematical programming. In: Crouzeix JP, et al (eds.) Generalized Convexity, Generalized Monotonicity: Recent Results. pp. 357-372. Kluwer, Dordrecht (1998)

13. Mishra, SK, Rurda, NG: Higher-order generalized invexity and duality in mathematical programming. J Math Anal Appl. 247, 173-182 (2000). doi:10.1006/jmaa.2000.6842

14. Chen, XH: Higher-order symmetric duality in nondifferentiable multiobjective programming problems. J Math Anal Appl. 290, 423-435 (2004). doi:10.1016/j.jmaa.2003.10.004

15. Mishra, SK: Second order symmetric duality in mathematical programming with F-convexity. Eur J Oper Res. 127, 507-518 (2000). doi:10.1016/S0377-2217(99)00334-3

16. Craven, BD: Lagrange conditions and quasiduality. Bull Austral Math Soc. 16, 325-339 (1977). doi:10.1017/ S0004972700023431

17. Hou, SH, Yang, XM: On second-order symmetric duality in nondifferentiable programming. J Math Anal Appl. 255 491-498 (2001). doi:10.1006/jmaa.2000.7242

18. Mishra, SK: Nondifferentiable higher-order symmetric duality in mathematical programming with generalized invexity. Eur J Oper Res. 167, 28-34 (2005). doi:10.1016/j.ejor.2004.02.024

19. Yang, $\mathrm{XM}$, Yang, $\mathrm{XQ}$, Teo, KL, Hou, SH: Second order symmetric duality in nondifferentiable multiobjective programming with F-convexity. Eur J Oper Res. 164, 406-416 (2005). doi:10.1016/j.ejor.2003.04.007

doi:10.1186/1029-242X-2012-142

Cite this article as: Ying: Higher-order symmetric duality for a class of multiobjective fractional programming problems. Journal of Inequalities and Applications 2012 2012:142.

\section{Submit your manuscript to a SpringerOpen ${ }^{\odot}$ journal and benefit from:}

- Convenient online submission

Rigorous peer review

- Immediate publication on acceptance

- Open access: articles freely available online

- High visibility within the field

- Retaining the copyright to your article

Submit your next manuscript at $\gg$ springeropen.com 